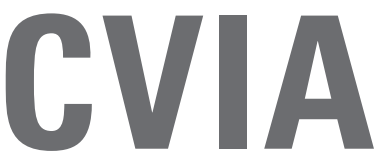

EDITORIAL

pISSN 2508-707X / elSSN 2508-7088 https://doi.org/10.22468/cvia.2016.00108 CVIA 2017;1 (1):2-3

CrossMark \& click for updates

Received: November 9, 2016

Revised: November 11, 2016

Accepted: November 11, 2016

Corresponding author

Tae Hoon Kim, MD, PhD

Department of Radiology,

Gangnam Severance Hospital,

211 Eonju-ro, Gangnam-gu,

Seoul 06273, Korea

Tel: 82-2-2019-3510

Fax: 82-2-3462-5472

E-mail: thkim1@yuhs.ac

\section{Cardiovascular Imaging Asia: A New Era in the Asian Society of Cardiovascular Imaging}

\author{
Tae Hoon Kim \\ Department of Radiology and Research Institute of Radiological Science, Gangnam Severance \\ Hospital, Yonsei University Health System, Seoul, Korea
}

\begin{abstract}
The Asian Society of Cardiovascular Imaging (ASCI) is launching its own ASCI journal in 2017, Cardiovascular Imaging Asia (CVIA). The journal is published with an open access format, four times a year in January, April, July, and October. The CVIA will cover all aspects of technical and clinical communication on cardiovascular diseases and its related imaging sciences. Original research, review articles, pictorial essays, and a few selected case reports with magnetic resonance imaging, computed tomography, X-ray imaging, interventional imaging, applications in nuclear cardiology and echocardiography, and any combination of these techniques will be included. The CVIA will be a leading journal in the field of cardiovascular imaging in Asia and will motivate the members of the ASCI to promote scientific activities.
\end{abstract}

Key words Heart · Imaging Society · Journal.
Scientific society is enhanced by the constant communication and accumulation of research outcomes performed by the community members. Many scientific societies, therefore, try to publish the valuable outcomes of their scientific and research activities based on global standards. In the field of cardiovascular imaging, many societies have published official journals, such as Circulation: Cardiovascular Imaging (http://circimaging.ahajournals.org) or International Journal of Cardiovascular Imaging (http://www.springer.com/medicine/cardiology/journal/10554).

The Asian Society of Cardiovascular Imaging (ASCI) was established under the endorsement of the Asian countries Japan, Singapore, China, Hong Kong, Taiwan, Vietnam, the Philippines, and Korea in April 2006, and later by Oceanian countries [1]. The ASCI has held annual meetings since 2007, and the 10th annual meeting of the ASCI was held in Singapore (August 4 to 6, 2016, Grand Copthorne Waterfront Singapore, Singapore) in 2016. The ASCI has published scientific papers to promote the scientific activities of the society members in collaboration with International Journal of Cardiovascular Imaging, which is the official journal of the North American Society for Cardiovascular Imaging, as an ASCI special issue since 2009.

The first ASCI special issue was published in April 2009, and these series continued publication until the final issue on June

(c) This is an Open Access article distributed under the terms of the Creative Commons Attribution Non-Commercial License (http://creativecommons.org/licenses/bync/3.0) which permits unrestricted non-commercial use, distribution, and reproduction in any medium, provided the original work is properly cited.
2016, consisting of 165 papers that have provided scientific motivation to the society members. Fourteen ASCI special issues were published with the generous support of Dr. Johan H.C. Reiber, the Editor-in-Chief at International Journal of Cardiovascular Imaging, and his colleagues, Dr. Yeon Hyeon Choe (2009-2013) and Dr. Tae Hoon Kim (2014-2016), who served as the guest Editors-in-Chief of the special issues, respectively. Despite the important roles of the ASCI special issues in the society, Dr. Yeon Hyeon Choe (Samsung Medical Center, Seoul, Korea), who was the current President of the ASCI, put forward a proposal to launch a new ASCI journal. The motivation for this new journal is based on the growth of the ASCI over the past 10 years and it intended to present the ASCI as a leader in the field of cardiovascular imaging in Asia. Although the members of the ASCI have published many valuable papers through the ASCI special issues, the ASCI members continue to demand a unique ASCI journal.

After many prudent discussions and debates on the current and future academic goals of the ASCI, the Executive Members of the ASCI finally decided to launch a new ASCI journal, Cardiovascular Imaging Asia (CVIA). The CVIA will be published with an open access format, four times a year in January, April, July, and October. The aims of the journal produce and propagate knowledge on cardiovascular imaging and its related sciences. Unique feature of the articles published in the journal will be the reflection of global trends in the cardiovascular im- 
aging field combined with an Asian perspective. The CVIA is freely available in electronic format at http://www.asci-cvia.org or http://www.e-cvia.org. All articles can be freely distributed and non-commercially used under the Creative Commons licenses (http://creativecommons.org/licenses/by/4.0), provided that the original works are properly cited.

The CVIA publishes all aspects of technical and clinical communication on cardiovascular diseases, encompassing original researches, review articles, pictorial essays with intuitive educational materials, and a few selected case reports. The journal will cover magnetic resonance imaging, computed tomography, Xray imaging, interventional imaging, applications in nuclear cardiology and echocardiography, and any combination of these techniques. The targets of the journal are physicians and other healthcare practitioners including cardiologists, radiologists, nuclear medicine physicians, pathologists, or cardiothoracic surgeons. The journal is also directed toward medical scientists, engineers, informatics specialists working on the development of imaging tools and/or contrast agents, and representatives from industries, organizations, and governments.

We have established the Editorial Board with the outstanding experts from Asian countries and the world in order to meet global standards in the process of journal publication. The journal is simultaneously published in print and on the official website (http://www.asci-cvia.org or http:///www.e-cvia.org). I am sure that the CVIA will be a leading journal in the field of cardiovascular imaging in Asia. On behalf of the members of the Editorial Board and publication staff, we look forward to receiving devoted contributions and a barrage of submissions from the members of the ASCI and international researchers.

\section{REFERENCE}

1. Choe YH. Launching of a new cardiovascular imaging society: the Asian Society of Cardiovascular Imaging. Int J Cardiovasc Imaging 2009;25 Suppl 1:3-7. 